\title{
Systematischer Index
}

Ordo Ganoidei ......... 1

A. Subord. Ch on d r o g a -

noidei ..... 14

1. Fam. Acipenseridae . . 14

A. Subfam. Acipenserinae . . 15

1. Gen. Acipenser L. . . . 15

a) Subgen. A cip e nser L. . 16

1. A. (A.) sturio L. . . . 18

2. " " ruthenus L. . 19

2a. " " " forma ru-

thena $\mathrm{L} . \ldots 21$

$2 b .$, , ruthenus forma kamensis Lovetzky ...221

3. " n naccarii Bp. . . 22

4. " " güldenstădti $r$. Brandt .....23

4a. \# ” güldenstădti forma güldenstådti F. Brandt 25

4b. , ॠ güldenstădti forma persica Borodin .....25

5. " " baerii F. Brandt 25

6. " " schrenckii F. Brandt . . . 25

7. „ " kikuchii Jord. \& Snyder ....27

8. "n multiscutatus Tanaka .....27

9. " " chinensis Gray . 27

10. " " transmontanus Richards. . . . . 29

11. " " medirostris Ayres . . . . . 29

12. " " fulvescens Raf. 30

13. " " brevirostrum Le Sueur . . . . 31

b) Subgen. Glad ostomus nom. nov. . . . . 31

14. A. (G.) stellatus Pall. . 32 14a. A. (G.) stellatus forma stellata Pall. . . 32

14b., " stellatus forma cyrensisL.S.Berg 32

c) Subgen. Lioniscus (Fitz. \& Heck.) Bp. . . 33

15. A. (L.) nudiventris Lovetzky . . . 33 3

2. Gen. Huso F. Brandt . . 34

1. H. huso (L.) . . . 35

2. "dauricus (Georgi) . 36 Bastarde

(ठ?) Acipenser (Acipenser) sturio L. $\times(\wp$ ?) A.(A.) guldenstădti $F$. Brandt 37

(O'?) Acipenser (Acipenser) ruthenus $\mathbf{L} . \times(Q$ ?) $\mathbf{A}$. (A). galdenstaddti $\mathrm{F}$. Brandt . . . . . . 37

( ${ }^{\top}$ ?) Acipenser (Acjpenser) ruthenus $L . X(Q$ ?) A. (A.) baerii F. Braddt . 37

( ${ }^{\top}$ ?) Acipenser (Acipenser) ruthenus L. $\times($ ? ?) A. (Gladostomus) stellatus Pall....... 37

( $ठ ?)$ Acipenser (Acipenser) ruthenus $\mathrm{L}$. $\times(Q$ ? $) \mathrm{A}$. (Lioniscus)nudiventris Lovetzky . . . . . . 37

( ${ }^{\circ}$ ?) Acipenser (Acipenser) güldenstådti $F$. Brandt $\times(\mathrm{Q}$ ?) A. (Gladostomus) stellatus Pall. . . 37

(ठ'?) Acipenser (Acipenser) güldenstădti F. Brandt $X$ ( ? ?) A. (Lioniscus) nudiventris Lovetzky . 37 
(ठ̛?) Acipenser (Acipenser) güldenstãdti F. Brandt $X$ (Q?) Huso huso (L.) 37

(ठ?) Acipeuser (Acipenser) schrenckii F. Brandt $X$ (o?) Huso dauricus (Georgi) . . . . . . 39

(ठ?) Acipenser (Gladostomus) stellatus Pall. $X$ (o?) d. (Lioniscus) nudiventris Lovetzky . . 39

(ठ??) Acipenser (Gladostomus) stellatus Pall. $X$ (६?) Huso huso (L.) . . 39

(రొ?) Acipenser (Lioniscus) nudiventris Lovetzky $X$ (Q?) Huso huso (L.) . . 39

?(?) Acipenser (Acipenser) ruthenus L. $x$ (?) Huso buso (L.) . . . . . . 39

B.Subfam.Scaphirhynchinae 39 3. Gen. Scaphirhynchus Heck. 40 1. S. platorhynchus (Kaf.) + 41

2. " mexicanus Giltay ...42

3. " albus (S. Forb. \& R. E. Richurds.) . . . . . 42

4. Gen. Pseudoscaphirhynchus Nikol. . . . . . . 42

1. P. fedtschenkoi (K. Kessl.) 43

2. " hermanni (K. Kessl.) . . 43

3. " kaufmauni (K. Kessl.) . 44

2. Fam. Polyodontidae . . 45

1. Gen. Polyodon (Lac.) Bl. \& J. G. Schn. . . . . . . 45

1. P. spathula (Walbaum) . 46
2. Gen. Psephurus Günther. . 46

1. P. gladius (Marts.) . . . 47

B. Subord. R h om bogano-

$$
\text { idei . . . . . } 47
$$

1. Fam. Lepisosteidae . . . 48

1. Gen. Lepisosteus Lac. . . 48

a) Subgen. Le pi s o ste u sac. 49 1. L. (L.) osseus (L.) . . 49 1a. " $" \quad$ osseus (L.) 50 Ib. " " $"$ treculii

(A. Dum.) 50

b) Subgen. Cylindrosteus

Raf. . . . . . . . . so

2. L. (C.) platostomus Raf. 51

2a. " " " platostomus

2b. " " " castelnaudii

A. Dum. . . 51

c) Subgen. Litholepis Raf. 52

3. L. (L.) tristoecbus (Bl. \& J.G.Schn.) 52

3a. " " " tristoechus (Bl. \& J. G. Schn.) 53

3b. " "tristoechus tropicus (Gill) . . 53

3c. " " $"$ megalops (H. W. Fowler) 53

Lepisosteus sinensis (Bleeker) 54

C. Subord.Cy clog a n oid $\theta$ i 54

1. Fam. Amiidae ...... 54

1. Gen. Amia L. . . . . . 55

1. A. calva L. . . . . . 56

Ompax spatuloides Cast. . . . 56 